\title{
What Are the Limits of Endoscopic Sinus Surgery?: The Expanded Endonasal Approach to the Skull Base
}

\author{
Carl H. Snyderman, ${ }^{1}$ Harshita Pant, ${ }^{1}$ Ricardo L. Carrau, ${ }^{1}$ Daniel Prevedello, ${ }^{2}$ \\ Paul Gardner ${ }^{2}$ and Amin B. Kassam ${ }^{3}$ \\ ${ }^{1}$ Department of Otolaryngology, University of Pittsburgh, Pennsylvania, USA \\ ${ }^{2}$ Department of Neurosurgery, University of Pittsburgh, Pennsylvania, USA \\ ${ }^{3}$ Brain Tumor Center and Neuroscience Institute, Saint John's Health Center and \\ John Wayne Cancer Institute, California, USA
}

(Received for publication on March 5, 2009)

(Revised for publication on March 30, 2009)

(Accepted for publication on April 16, 2009)

\begin{abstract}
The advent of endoscopic technologies and techniques has expanded the limits of conventional endoscopic sinus surgery. The expanded endonasal approach describes a series of surgical modules in the sagittal and coronal planes that allow surgical access to the entire ventral skull base. The sagittal plane extends from the frontal sinus to the second cervical vertebra. The coronal plane extends from the midline to the roof of the orbit, the floor of the middle cranial fossa, and the jugular foramen. Key principles of endonasal skull base surgery are choosing a surgical corridor that minimizes the need for neural and vascular manipulation, team surgery, use of the endoscope to enhance visualization, and bimanual tumor dissection under direct visualization. Particular challenges of the expanded endonasal approach are identification of anatomical structures using unfamiliar landmarks, hemostasis, and dural reconstruction. Over the last decade with more than 1000 completely endonasal skull base surgeries, we have demonstrated that endoscopic endonasal surgery of the skull base can be performed with minimal morbidity and mortality. The introduction of the septal mucosal flap for dural reconstruction has decreased the incidence of postoperative cerebrospinal fluid leaks to less than 5\%. Early data suggests that oncological outcomes for malignant sinonasal tumors with skull base involvement are comparable to conventional techniques. Proper training in endonasal surgical techniques is essential to prevent unnecessary morbidity and achieve good outcomes. (Keio J Med 58 (3) : 152-160, September 2009)
\end{abstract}

Keywords: endoscopic sinus surgery, skull base, cranial base, sinonasal neoplasms

\section{Introduction}

Endoscopic surgical techniques have transformed all of the surgical specialties. In otolaryngology, endoscopic sinus surgery is well established for the treatment of inflammatory sinus disease as well as benign and selected malignant sinonasal tumors. Endoscopic techniques have also been applied to the treatment of cranial base pathology, most commonly cerebrospinal fluid leaks and pituitary tumors. ${ }^{1}$ Over the last decade, we have demonstrated that the entire ventral skull base is accessible using an endonasal approach. This is termed the "expanded endonasal approach (EEA)" and provides access to the anterior, middle, and posterior cranial fossae. ${ }^{2-4}$

The limitations of the EEA have yet to be established. Initially, the development of endonasal skull base surgery was limited by available technology and anatomical knowledge of the skull base from an endonasal perspec-

Presented at the 1599th Meeting of The Keio Medical Society in Tokyo, November 21, 2008.

Reprint requests to: Carl H. Snyderman, MD, Department of Otolaryngology, University of Pittsburgh, Suite 500, Eye \& Ear Institute, 200 Lothrop St, Pittsburgh, PA15213, USA, Tel: +1-412-647-8186, Fax: +1- 412-647-2080, Email: snydermanch@upmc.edu 


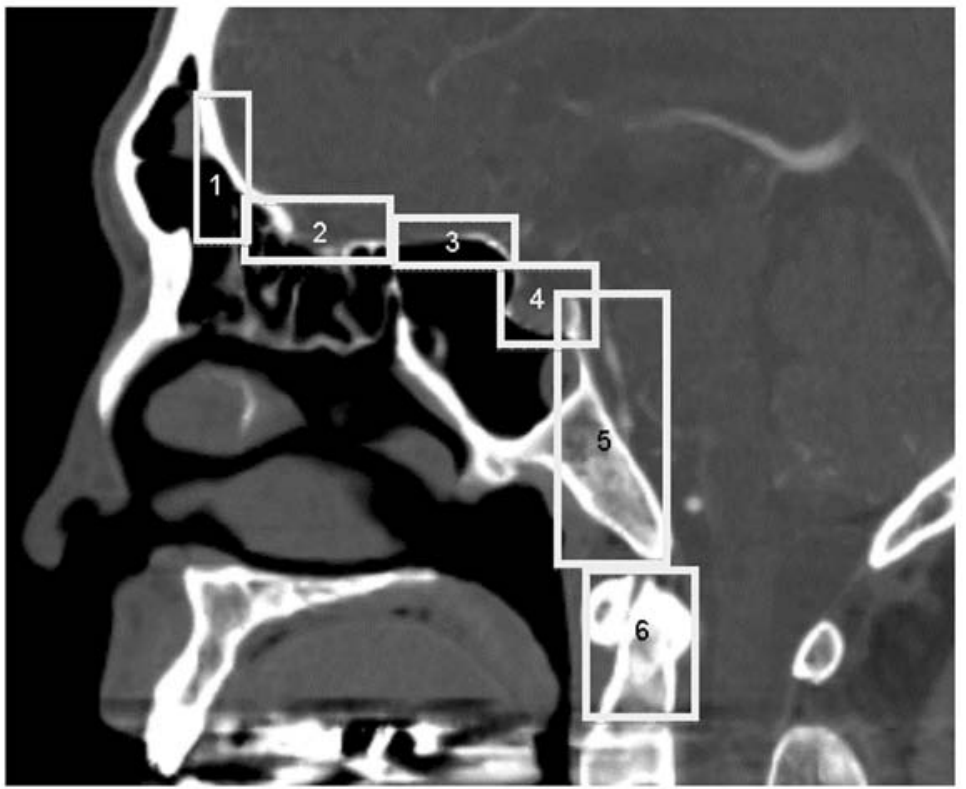

Fig. 1 Schematic depiction of the sagittal plane module on a computed-tomography (CT) scan of the skull base. The various endonasal surgical approaches are numbered as follows; (1) transfrontal (2) transcribriform, (3) transplanum, (4) transsphenoid, (5) transclival and (6) transodontoid.

tive. Necessary technology included instruments adapted for intranasal use such as dissecting instruments and powered instrumentation (drill, ultrasonic aspirator, and microdebrider), intraoperative navigational devices, hemostatic materials and devices (bipolar forceps), and materials for dural reconstruction. Although much was known about skull base anatomy, there was little knowledge about key relationships of neurovascular structures from the endonasal perspective, in particular landmarks for the different segments of the internal carotid artery (ICA). Other potential limitations of endoscopic skull base surgery include the nature of the pathology (location, diagnosis, vascularity), the patient (age, medical comorbidities), the surgeon (training or expertise), and institution (resources).

\section{Location and Surgical Access}

The primary factor limiting EEA is the location of the pathology relative to important neural and vascular structures. The key principle of skull base surgery is choosing the best pathway to the pathology. For many tumors, the endonasal corridor provides the most direct access with the least manipulation of neural and vascular structures. For example, the endonasal corridor provides a direct pathway to an olfactory groove meningioma without the need for brain retraction. Conversely, a tumor lateral to the optic nerve is best approached via another approach. Large tumors may require a combination of external and endonasal approaches.

The EEA consists of multiple modules that are oriented in sagittal and coronal planes., ${ }^{2,3}$ Sagittal plane modules extend from the frontal sinus to the second cervical vertebra (Fig. 1). ${ }^{1}$ The transfrontal module provides access to the floor and posterior wall of the frontal sinus. Examples of lesions found in this area include inflammatory sinus disease, erosive mucocoeles, frontal osteomas, and nasal dermoids. The transfrontal approach is the anterior limit of an endonasal "craniofacial resection" for malignant sinonasal neoplasms. ${ }^{2}$ The transcribriform module extends from the crista galli to the planum sphenoidale and across the roof of the ethmoid sinuses (fovea ethmoidalis) to the orbital roof. This module is most often used for sinonasal malignancies and olfactory groove meningiomas. ${ }^{3}$ The transplanum module provides access to suprasellar lesions such as pituitary tumors with extrasellar extension and craniopharyngiomas. It is limited posterolaterally by the optic canals. ${ }^{4}$ An example of a lesion (olfactory groove schwannoma) requiring a transcribriform and transplanum approach is depicted in Figure 2. The transsellar approach is the standard approach for pituitary pathology but may be combined with other approaches for tumors with extrasellar extension. It is limited laterally by the cavernous ICA. ${ }^{5}$ The transclival approach spans the sphenoid bone and clivus from the posterior clinoids to the foramen magnum and can be subdivided into superior, middle, and inferior transclival approaches (Fig. 3). The superior transclival approach requires a transposition of the pituitary gland superiorly with preservation of the pituitary stalk. Typical clival lesions include chordomas and meningiomas. ${ }^{6,7}$ The transodontoid approach provides access to 

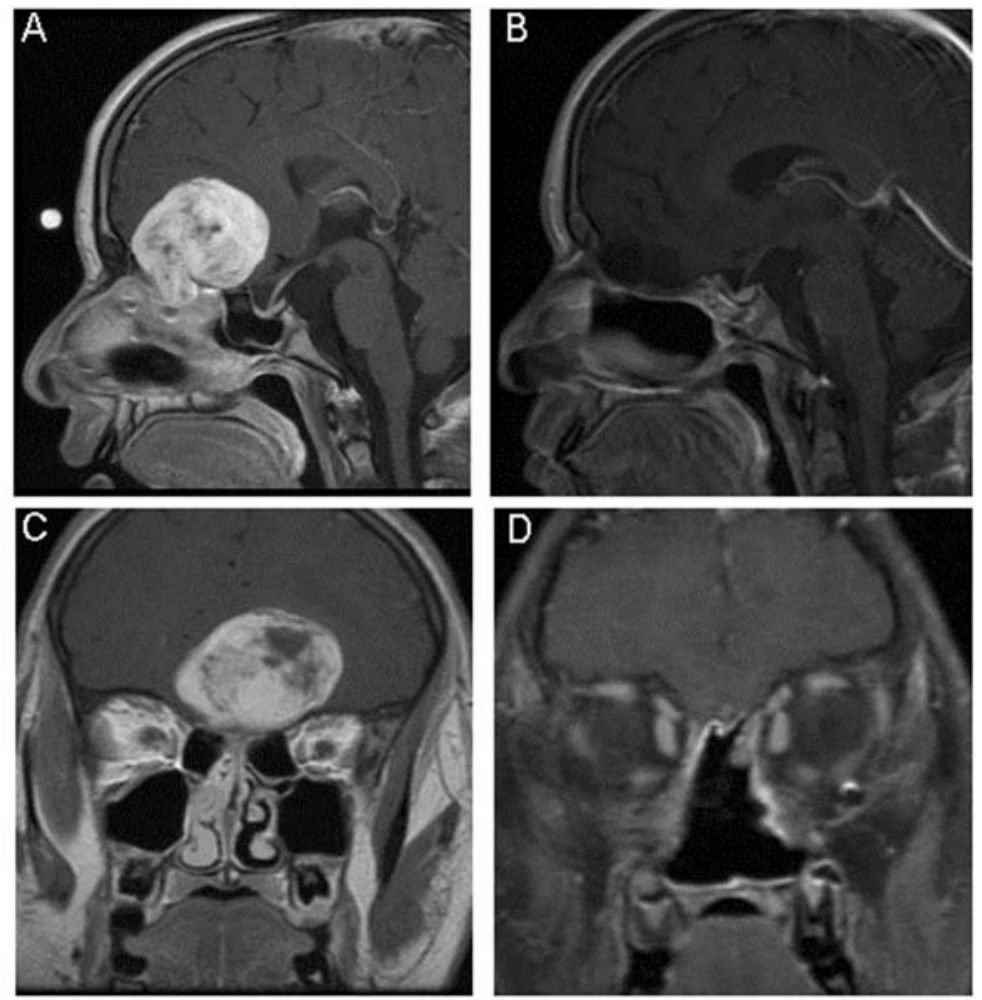

Fig. 2 Magnetic resonance imaging (MRI) of an olfactory groove schwannoma in a teenage male. An endoscopic completely endonasal transcribriform and tranplanum approach was utilized to remove this tumor. Figures A and B represent preoperative scans and figures $\mathrm{C}$ and $\mathrm{D}$ postoperative scans.
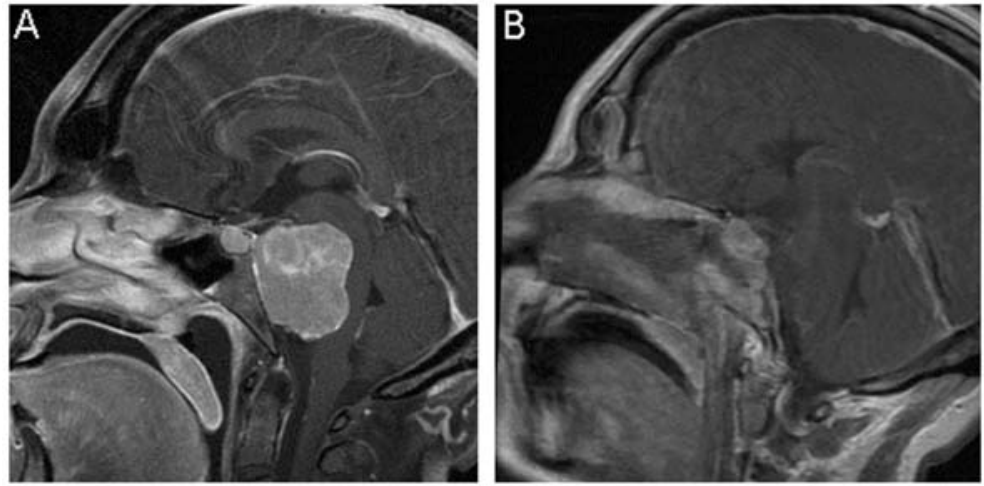

Fig. 3 Preoperative (A) and postoperative (B) MRI of a patient with clival meningioma.

the upper cervical spine $(\mathrm{C} 1$ and $\mathrm{C} 2)$ and foramen magnum. ${ }^{5}$ Rheumatoid degeneration of the upper cervical spine with pannus formation is associated with basilar invagination of the spine and compression of the brainstem (Fig. 4). Removal of the ring of $\mathrm{C} 1$ and odontoid decompresses the brainstem anteriorly but still requires stabilization of the spine posteriorly. A transodontoid approach is also used for access to spinal tumors at the level of the foramen magnum. This approach is limited laterally by the parapharyngeal ICA and the vertebral arteries. The inferior limit is defined by the nasopalatine line, a line that is tangential to the inferior edge of the nasal bones and the posterior edge of the hard palate. ${ }^{6,8}$ The maximal amount of bone that can be removed transnasally is delimited by where a projection of the line crosses the cervical spine.

EEA approaches in the coronal plane are divided into anterior, middle, and posterior coronal planes corre- 

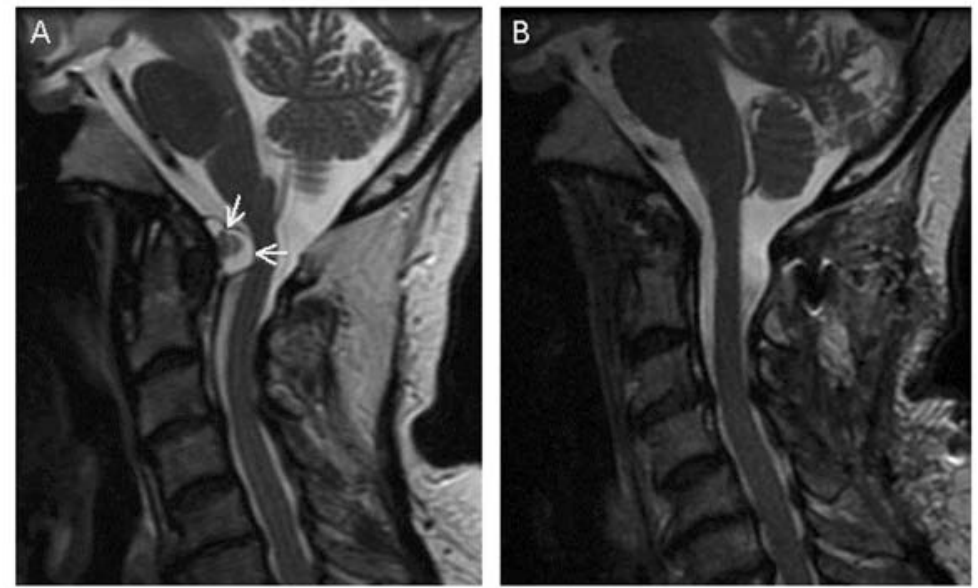

Fig. 4 MRI of the cervical spine in the sagittal plane showing compression of the lower brainstem and cervical spine from a rheumatoid arthritis pannus (white arrows). The pannus was removed via an endoscopic transodontoid approach. Preoperative (A) and post-operative (B) scans.

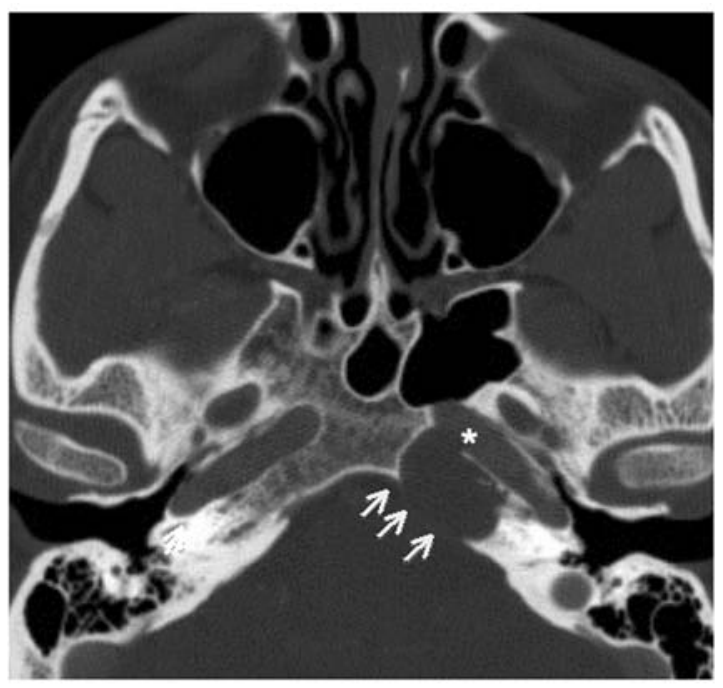

Fig. 5 An axial CT scan showing an expansile cholesterol granuloma (margins marked with white arrows) situated medial to the petrous internal carotid artery $(*)$. This was drained via an endoscopic transpterygoid and transphenoid approach.

sponding to the corresponding cranial fossae. ${ }^{4}$ The anterior coronal plane consists of supraorbital and transorbital approaches. The supraorbital approach requires removal of the medial wall of the orbit and displacement of the orbital tissues to visualize the orbital roof. This approach is used for benign fibro-osseous lesions involving orbital walls and to obtain lateral margins with a transcribriform or transplanum approach. The mid-plane of the orbital roof can be reliably reached with this approach. The transorbital approach is used for intraconal lesions that are inferior and medial to the optic nerve such as hemangiomas and schwannomas. Access is between the inferior and medial rectus muscles with preservation of extraocular muscle function.

The middle coronal plane extends from the sphenoid sinus across the floor of the middle cranial fossa superior to the petrous ICA. ${ }^{9}$ An important landmark for localization of the petrous ICA is the pterygoid or vidian canal. ${ }^{6}$ The vidian artery can be followed to the second genu of the ICA. ${ }^{10}$ The medial petrous apex can be approached at the level of the clival recess between the mid-clival bone and the paraclival segment of the ICA. ${ }^{11}$ Expansile lesions of the petrous apex such as cholesterol granulomas are well-suited to this approach (Fig. 5). Meckel's cave and the cavernous sinus can be approached lateral to the paraclival segment and superior to the petrous segment of the ICA between the vidian nerve and second division of the trigeminal nerve, V2, leading to the foramen rotundum. This requires a transpterygoid approach with full access to the lateral recess of the sphenoid sinus. Meningiomas and schwannomas may require this approach as well as malignant tumors with perineural extension along V2 (squamous cell carcinoma, adenoid cystic carcinoma). Further laterally, tumors of the infratemporal fossa (mandibular nerve (V3) schwannoma) may be approached. This requires a medial maxillectomy with Denker's maxillotomy (anteromedial maxillectomy) for full access to the posterolateral wall of the maxillary sinus. The lateral pterygoid plate is a useful landmark for locating foramen ovale, where V3 tumors are located.

The posterior coronal plane extends from the foramen magnum across the occipital condyle and hypoglossal canal to the jugular foramen. ${ }^{3}$ Chondrosarcomas, meningiomas, chordomas, are the most common tumors in this location. Resection of the Eustachian tube is necessary to gain full access to the infrapetrous area and is an im- 


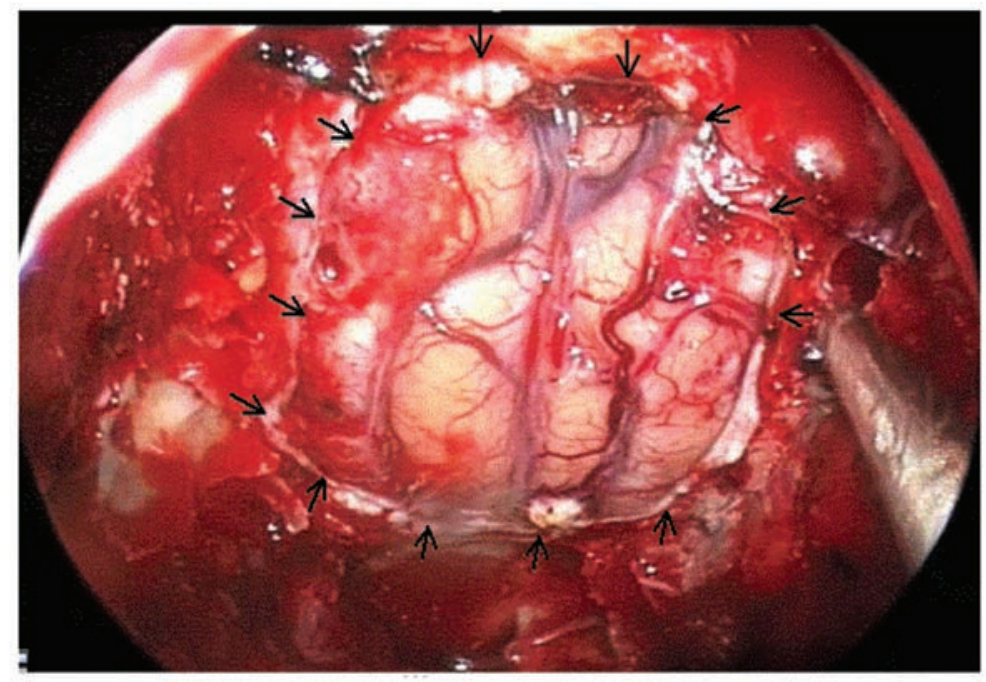

Fig. 6 An endoscopic view of the surgical dural defect (margins delineated with black arrows) after an endoscopic transcribriform approach for excision of an esthesioneuroblastoma. The ventral surface of the frontal lobes is evident beyond the defect.

portant landmark for the parapharyngeal ICA. An infrapetrous approach can also be used to access pathology of the petrous apex that cannot be reached using a medial approach. ${ }^{9}$

\section{Oncological Resection}

A large variety of malignant sinonasal tumors can involve the skull base. For those tumors considered operable, a craniofacial resection with postoperative radiation $+/$ - chemotherapy is considered the gold standard. This has been well established, in particular, for esthesioneuroblastomas where skull base erosion and dural invasion are typical features. One criticism of endonasal surgery for sinonasal malignancies is that it does not respect oncological principles. When properly performed, oncological principles do not need to be sacrificed to achieve a complete resection with clear margins. ${ }^{12}$ Our collective experience with other head and neck neoplasms demonstrates that piecemeal resection of the tumor does not compromise results as long as the final margins are clear (endoscopic resection of inverting papilloma, transoral supraglottic laryngectomy, Moh's technique for skin cancer). Although a sinonasal neoplasm may fill the nasal cavity, the area of tumor invasion may be quite limited. In the case of esthesioneuroblastoma, the intranasal component of the tumor is debulked and the bone around the periphery of the tumor is exposed and removed (floor of the frontal sinuses and crista galli anteriorly, medial orbit and fovea ethmoidalis laterally, and planum sphenoidale posteriorly). ${ }^{2}$ The dura is then incised around the periphery of the tumor and dissected free from the surface of the brain with inclusion of the olfactory bulbs and tracts. Thus, the area of skull base involvement is removed en bloc and clear margins are confirmed with frozen section analysis (Fig. 6).

Although the literature is quite sparse and follow up is limited, the early results of completely endoscopic endonasal resection of olfactory neuroblastomas are very encouraging. ${ }^{13}$ Until larger series are published with extended follow up ( $>5$ years), a complete oncological resection as outlined here should be performed.

\section{Prevention of Complications}

The most important innovation in skull base surgery for the prevention of complications has been the introduction of true team surgery. Unlike other team surgeries that employ sequential teams of surgeons, endonasal skull base surgery requires the participation of both surgical specialties throughout the surgery. The benefits of team surgery are myriad and include improved visualization, increased operative efficiency, improved decision making and problem solving, and modulation of individual enthusiasm. Endoscopic visualization provided by an experienced surgeon is a very dynamic process in which constant adjustments are made to provide room for instrumentation and provide the best view. This assumes critical importance when there is a vascular emergency.

There are a number of technological challenges in performing endonasal skull base surgery. Foremost among these are hemostasis and dural repair. ${ }^{6,12,14}$ Strategies for minimizing intraoperative blood loss include preoperative embolization when feasible, surgical ligation of vessels at the periphery of the tumor (anterior and posterior ethmoidal arteries), and removal of the bone and dural base of the tumor. The use of a diamond drill for 

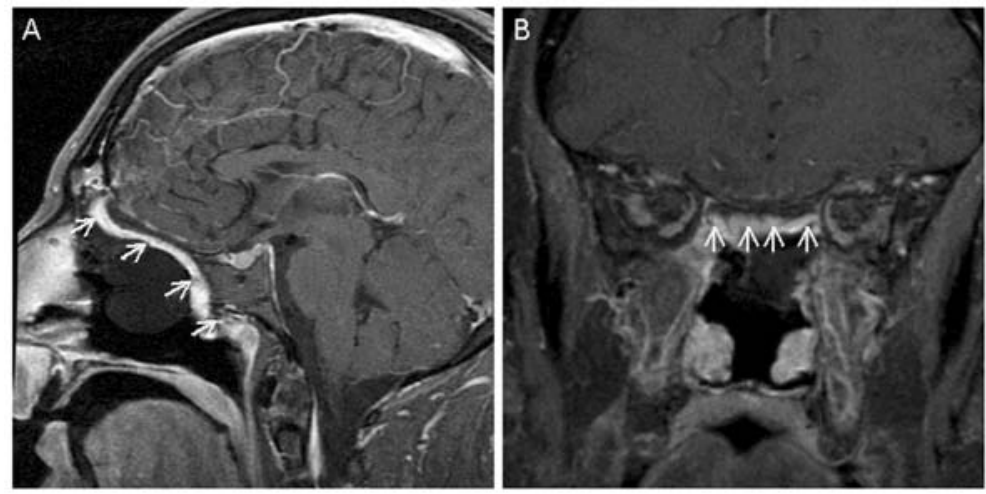

Fig. 7 Postoperative MRI of the skull base in the sagittal (A) and coronal plane (B) of a patient that underwent endoscopic transcribriform resection of an esthesioneuroblastoma. The extent of skull base defect repaired using a Hadad-Bassagasteguy nasoseptal flap is demonstrated. A foley catheter balloon can be seen in place that is used to provide support for the flap in the immediate post-operative period.

bone removal provides a hemostatic effect. The most important instrument is the endoscopic bipolar electrocautery device with exchangeable tips for different bleeding situatons. A variety of hemostatic materials are available for direct application to bleeding sites. Hot water irrigation $\left(40^{\circ} \mathrm{C}\right)$ with saline is a useful adjunct for diffuse oozing from sinus mucosa as well as intracranially.

Reconstruction of large dural defects has been one of the greatest challenges of endonasal skull base surgery. Our initial experience was plagued by an unacceptable level of postoperative cerebrospinal fluid (CSF) leaks. This has been solved with a combination of techniques including inlay and onlay fascial grafts, direct suturing of dural grafts, fat grafts, the introduction of vascularized pedicled flaps, and intranasal support with a balloon catheter. ${ }^{15,16-20}$ Our preferred technique is a septal mucosal flap pedicled on the posterior septal artery from the sphenopalatine foramen. This provides complete coverage of a craniofacial defect from the frontal sinuses to the planum sphenoidale and from orbit to orbit (Fig. 7). When this tissue is not available, a pericranial scalp flap (without a craniotomy) or a temporoparietal fascial flap can be used. ${ }^{14}$ In a series of 150 consecutive cases with intraoperative CSF leak, the incidence of postoperative leaks was $4 \%$ (unpublished data).

\section{Dealing with Complications}

A frequent concern is whether endoscopic endonasal techniques are sufficient to deal with a major vascular injury such as injury to the ICA. An experienced team of surgeons can maintain visualization and gain control with bipolar electrocautery, application of hemostatic materials, and focal packing. Indiscriminate packing is to be avoided since blood may track intracranially. If necessary, aneurysm clamps can be placed across an in- jured vessel. Once intraoperative control is achieved, angiography is performed with possible sacrifice of the vessel.

Infectious complications are surprisingly rare with endonasal skull base surgery, with an incidence of bacterial meningitis of $1-2 \%$. This is attributed to minimal bacterial colonization of the nasal cavity and sinuses (compared to the oral cavity), prophylactic perioperative antibiotics, frequent intraoperative irrigation, skull base reconstruction with vascularized flaps, and aggressive management of postoperative CSF leaks. Non-vascularized materials such as bone cement or titanium plates should be avoided due to the risk of infection.

Postoperative CSF leaks are managed aggressively with endoscopic surgical repair. In almost all cases, the repair can be augmented with a small fascial or fat graft or with repositioning of the mucosal flap. A lumbar spinal drain is reserved for high-risk repairs (large dural defects, high-flow leaks, prior surgery or radiation therapy) or recurrent CSF leaks.

\section{Staging of Surgery}

Although endoscopic techniques have many potential advantages, duration of surgery is not always one of them. In some cases, it may be more expedient to perform an open approach when outcomes are comparable. Long surgeries increase risks for the patient and are difficult for the surgeon. Staging of surgery should be considered when extensive bone removal is required to gain access to the tumor, especially if there is significant blood loss. Removal of the bone and cauterization of the dural base decreases intraoperative blood loss during the second stage. Excessive blood loss can create a coagulopathic state that is more problematic with intradural dissection. Large juvenile nasopharyngeal angiofibromas with skull base erosion and intracranial extension may 

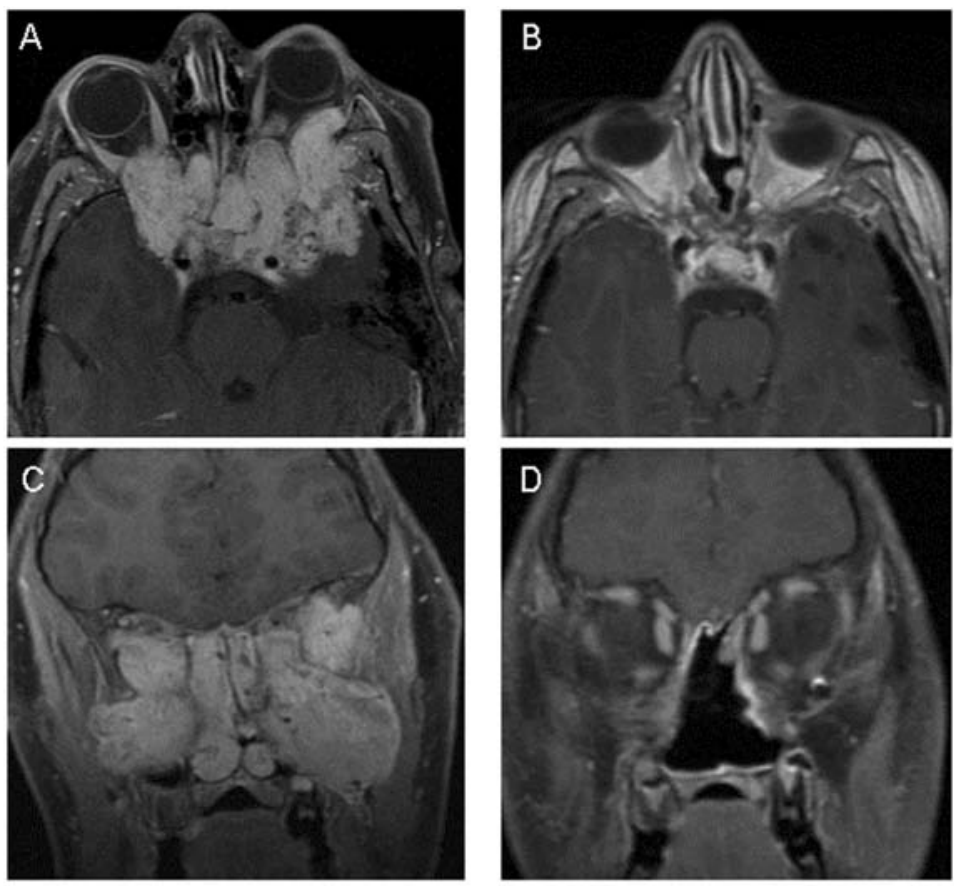

Fig. 8 MRI showing preoperative (A, B) and postoperative (C, D) scans of a patient with a giant juvenile nasopharyngeal angiofibroma. This tumor was removed using an entirely endonasal endoscopic approach and required 3 operations for complete excision.

receive significant blood supply from the ICA and remain highly vascularized despite preoperative embolization of the external carotid arteries. Other factors to be considered in staging surgery include the duration of surgery where late in the day, surgeon fatigue and lack of availability of experienced operating room personnel may compromise outcomes. In rare cases, the entire tumor may not be accessed and staging the procedure could enable complete resection due to movement of the residual tumor into the surgical field. With large intracranial tumors, determination of tumor margins and assessment of the extent of resection can be difficult due to shifting of the tumor. Image-guided navigation can be updated at a second stage or with the use of intraoperative imaging.

\section{Special Patient Populations: Pediatric Patients}

Decreased morbidity, faster recovery, and greater patient acceptance make the EEA especially suitable for pediatric patients..$^{21-23}$ Despite smaller nares and nasal cavities, most patients older than 4 years have sufficient room for endoscopic surgery. If not, a transoral approach (midfacial degloving) can be used to access the nasal cavity. Decreased sinus pneumatization in the pediatric population can make identification of normal anatomical structures more difficult. Applications of EEA in the pediatric population include biopsy for diagnosis, definitive treatment of benign neoplasms (including pituitary adenoma, juvenile nasopharyngioma and craniopharyngioma) and down-staging of high-grade malignancies such as rhabdomyosarcoma.

Juvenile nasopharyngeal angiofibroma is unique to the pediatric population and poses a particular challenge because of their increased vascularity. Even large tumors with skull base involvement can be successfully managed using endoscopic techniques and tumors medial and lateral to the ICA can be managed using one approach. Staging of the surgery is often necessary due to increased blood loss (Fig. 8). Surgical removal of large tumors is performed by dividing the tumor into several segments corresponding to its blood supply. Each vascular segment is excised sequentially and the intracranial extension is excised last. The endoscopic approach in pediatric patients minimizes morbidity and potentially avoids craniofacial growth abnormalities due to the disruption of growth centers.

\section{Training}

Proper training in endonasal surgical techniques is essential to achieve optimum surgical and oncological outcomes and to avoid complications. ${ }^{24}$ Ideally, skull base surgeons should be trained in both, open and endoscopic, techniques so that the best surgical option can be offered to the patient for any given pathology. We have devised 
Table 1 Training levels for endonasal skull base surgery

\begin{tabular}{ll}
\hline \hline Level I & Sinus Surgery \\
Level II & Advanced sinus surgery \\
& Cerebrospinal fluid leak \\
& Intrasellar - sella, pituitary \\
Level III & Extrasellar - sella, pituitary \\
& Optic nerve decompression \\
& Intraorbital surgery \\
& Extradural skull base surgery \\
Level IV & Intradural skull base surgery \\
& A. With cortical cuff \\
& $\quad$ Transplanum \\
& $\quad$ Transcribriform \\
& Type I Craniopharyngioma \\
& Intradural skull base surgery \\
& B. Without cortical cuff \\
& $\quad$ Type II/III Craniopharyngioma \\
& $\quad$ Transclival, intradural \\
Level V & Coronal plane, carotid dissection \\
& Vascular surgery \\
\hline
\end{tabular}

a 5-level training program that is based on our own learning experience (Table 1). ${ }^{25,26}$ These levels incorporate anatomical complexity, technical difficulty, potential risk of neural and vascular injury, extent of intradural dissection and type of pathology. Thus, mastering a level before proceding to the next is recommended. This training program applies to all endonasal surgeons, irrespective of their specialty.

Level I consists of basic endoscopic sinus surgery. Level II incorporates advanced endoscopic sinus surgery and the basic skull base surgery including management of CSF leaks and small pituitary adenomas. This is where otolaryngologists and neurosurgeons may work together to build their team skills and master hemostatic and reconstructive techniques. Level III includes management of extradural skull base tumors and pituitary adenomas with extrasellar extension. Level IV procedures require intradural dissection and are among the most challenging cases. "Craniofacial resection" for sinonasal malignancies is included in Level IV due to the importance of adhering to oncological principles. If there is no cuff of brain tissue separating the margin of the tumor from cerebral vessels, an additional level of difficulty is assigned (Level IVb). Level V surgeries are less common in routine practice and involve dissection around the ICA in the coronal plane (infrapetrous approach). Management of vascular tumors and aneurysms are included in Level V.

\section{Resources}

Necessary resources include endonasal instrumentation, the operating theater, operating room personnel and institutional resources. Neurophysiologic monitoring of brain and nerve function is routinely performed to detect cerebral ischemia and prevent cranial nerve injury. Image guidance using fused CT and MRI scans is essential to navigate the "black box" of the skull base. Newer operating theaters incorporate a CT scanner so that image guidance can be updated intraoperatively to account for tumor shift and to assess completeness of resection. The operating theater is designed for endonasal endoscopic surgery and has multiple viewing monitors for individual surgeons and operating room staff so that all are aware of the status of the surgery. A telestration monitor facilitates communication between surgeons and is a useful teaching tool.

There are a number of obstacles to the development of endoscopic endonasal cranial base surgery. The biggest challenge is assembling a team of surgeons that are interested in working collaboratively and obtaining the necessary training. The surgical team should work together consistently to learn how to function as a team and develop expertise with hemostatic and dural reconstruction techniques. They should have a sufficient surgical volume to maintain their expertise. Institutional resources and billing issues may also limit development.

\section{Conclusions}

The entire ventral skull base can be accessed endonasally using completely endoscopic techniques. Surgical approaches begin in the sphenoid sinus as a reference and is classified into modules based on their orientation in the sagittal and coronal planes. Some of the major obstacles to endonasal brain surgery, including hemostasis and dural defect reconstruction, are being managed effectively, however, the techniques and materials used will continue to evolve. The limits of endonasal cranial base surgery have not been realized. The choice of a surgical approach depends on patient co-morbidities, tumor characteristics, and the skill and comfort level of the surgeons. If nerves or vessels need to be mobilized to reach the tumor, them an alternative approach should be considered. Relative contraindications to an endonasal approach include tumor involvement of superficial tissues, the need for vascular reconstruction and the duration of surgery. If sinusitis is identified, an intradural approach should not be undertaken until the infection has cleared.

Potential advantages of endoscopic endonasal cranial base surgery include the lack of external incision, decreased trauma to normal soft tissue and bone, improved visualization, increased access, improved outcomes, fewer complications, more rapid recovery, and decreased hospitalization and cost. Disadvantages include unfamiliar anatomy, a long learning curve, and technological limitations. Surgeons should be adequately trained in these procedures and have adequate experience, surgical 
volume, and resources before undertaking more challenging surgeries. The endonasal corridor is one more option for our patients with diseases of the cranial base and should be part of the surgical armamentarium of every cranial base surgeon.

\section{References}

1. Carrau RL, Kassam AB, Snyderman CH: Pituitary surgery. Otolaryngol Clin North Am 2001; 34: 1143-1155, ix

2. Kassam A, Snyderman CH, Mintz A, Gardner P, Carrau RL: Expanded endonasal approach: the rostrocaudal axis. Part I. Crista galli to the sella turcica. Neurosurg Focus 2005; 19: E3

3. Kassam A, Snyderman CH, Mintz A, Gardner P, Carrau RL: Expanded endonasal approach: the rostrocaudal axis. Part II. Posterior clinoids to the foramen magnum. Neurosurg Focus 2005; 19: E4

4. Kassam AB, Gardner P, Snyderman C, Mintz A, Carrau R: Expanded endonasal approach: fully endoscopic, completely transnasal approach to the middle third of the clivus, petrous bone, middle cranial fossa, and infratemporal fossa. Neurosurg Focus 2005; 19: E6

5. Mehta RP, Cueva RA, Brown JD, Fliss DM, Gil Z, Kassam AB, Rassekh CH, Schlosser RJ, Snyderman CH, Har-El G: What's new in skull base medicine and surgery? Skull Base Committee Report. Otolaryngol Head Neck Surg 2006; 135: 620-630

6. Kassam A, Snyderman CH, Carrau RL, Gardner P, Mintz A: Endoneurosurgical hemostasis techniques: lessons learned from 400 cases. Neurosurg Focus 2005; 19: E7

7. Stippler M, Gardner PA, Snyderman CH, Carrau RL, Prevedello DM, Kassam AB: Endoscopic endonasal approach for clival chordomas. Neurosurgery 2009; 64: 268-277; discussion 277-268

8. de Almeida JR, Zanation AM, Snyderman CH, Carrau RL, Prevedello DM, Gardner PA, Kassam AB: Defining the nasopalatine line: the limit for endonasal surgery of the spine. Laryngoscope 2009; 119: 239-244

9. Zanation AM, Snyderman CH, Carrau RL, Gardner PA, Prevedello DM, Kassam AB: Endoscopic endonasal surgery for petrous apex lesions. Laryngoscope 2009; 119: 19-25

10. Vescan AD, Snyderman CH, Carrau RL, Mintz A, Gardner P, Branstetter Bt, Kassam AB: Vidian canal: analysis and relationship to the internal carotid artery. Laryngoscope 2007; 117: 1338 $-1342$

11. Kassam A, Carrau RL, Snyderman CH, Gardner P, Mintz A: Evolution of reconstructive techniques following endoscopic expanded endonasal approaches. Neurosurg Focus 2005; 19: E8

12. Snyderman CH, Kassam AB, Carrau R, Mintz A: Endoscopic Reconstruction of Cranial Base Defects following Endonasal Skull Base Surgery. Skull Base 2007; 17: 73-78
13. Folbe A, Herzallah I, Duvvuri U, Bublik M, Sargi Z, Snyderman $\mathrm{CH}$, Carrau R, Casiano R: Endoscopic endonasal resection of esthesioneuroblastoma: A multicentre study. Am J Rhinol Allergy 2009; 23: 91-94

14. Zanation AM, Snyderman CH, Carrau RL, Kassam AB, Gardner PA, Prevedello DM: Minimally invasive endoscopic pericranial flap: a new method for endonasal skull base reconstruction. Laryngoscope 2009 ; 119: $13-18$

15. Kassam AB, Snyderman C, Gardner P, Carrau R, Spiro R: The expanded endonasal approach: a fully endoscopic transnasal approach and resection of the odontoid process: technical case report. Neurosurgery 2005; 57: E213; discussion E213

16. Snyderman CH, Kassam AB, Carrau R, Mintz A: Endoscopic approaches to the petrous apex. Operative Techniques in Otolaryngology 2006; 17: 168-173

17. Tosun F, Carrau RL, Snyderman CH, Kassam A, Celin S, Schaitkin B: Endonasal endoscopic repair of cerebrospinal fluid leaks of the sphenoid sinus. Arch Otolaryngol Head Neck Surg 2003; 129: $576-580$

18. Al-Nashar IS, Carrau RL, Herrera A, Snyderman CH: Endoscopic transnasal transpterygopalatine fossa approach to the lateral recess of the sphenoid sinus. Laryngoscope 2004; 114: 528-532

19. Hadad G, Bassagasteguy L, Carrau RL, Mataza JC, Kassam A, Snyderman CH, Mintz A: A novel reconstructive technique after endoscopic expanded endonasal approaches: vascular pedicle nasoseptal flap. Laryngoscope 2006; 116: $1882-1886$

20. Oliver CL, Hackman TG, Carrau RL, Snyderman CH, Kassam AB, Prevedello DM, Gardner P: Palatal Flap Modifications Allow Pedicled Reconstruction of the Skull Base. Laryngoscope 2008; 118: $2102-2106$

21. Zimmer LA, Hirsch BE, Kassam A, Horowitz M, Snyderman CH: Resection of a recurrent paraganglioma via an endoscopic transnasal approach to the jugular fossa. Otol Neurotol 2006; 27: 398 402

22. Kassam A, Thomas AJ, Snyderman C, Carrau R, Gardner P, Mintz A, Kanaan H, Horowitz M, Pollack IF: Fully endoscopic expanded endonasal approach treating skull base lesions in pediatric patients. J Neurosurg 2007; 106: 75-86

23. Pirris SM, Pollack IF, Snyderman CH, Carrau RL, Spiro RM, Tyler-Kabara E, Kassam AB: Corridor surgery: the current paradigm for skull base surgery. Childs Nerv Syst 2007; 23: 377-384

24. Snyderman C, Kassam A, Carrau R, Mintz A, Gardner P, Prevedello DM: Acquisition of surgical skills for endonasal skull base surgery: a training program. Laryngoscope $2007 ; \mathbf{1 1 7}$ : 699-705

25. Kassam AB, Gardner P, Snyderman C, Carrau R, Zimmer LA, Hirsch B, Mintz A: Endoscopic,e expanded endonasal approach to the jugular foramen. Operative Techniques in Neurosurgery: Management of Jugular Foramen Tumors 2005; 8: 35-41

26. Snyderman C, Carrau R, Kassam A: Who is the skull base surgeon of the future? Skull Base 2007; 17: 353-355 